\title{
Coronavirus Disease (COVID-19) Testing in Asymptomatic Patients Planned for Day Care Systemic Therapy: A Tertiary Cancer Center Experience
}

\author{
Jerin Ovett ${ }^{1}$ Manikandan Dhanushkodi ${ }^{1}$ Venkatraman Radhakrishnan ${ }^{1} \quad$ Nikita Mehra ${ }^{1}$ \\ Jayachandran Perumal Kalaiarasi ${ }^{1}$ Gangothri Selvarajan¹ Arun Kumar Rajan¹ \\ Parathan Karunakaran ${ }^{1}$ Sivasree Kesana ${ }^{1}$ Tenali Gnana Sagar ${ }^{1}$
}

${ }^{1}$ Department of Medical Oncology, Cancer Institute (WIA), Chennai, Tamil Nadu, India

Ind J Med Paediatr Oncol 2021;42:11-14.
Address for correspondence Manikandan Dhanushkodi, MD, DM, DNB, Department of Medical, Oncology, Cancer, Institute (WIA), 38, Sardar, Patel Road, Chennai, Tamil Nadu, 600036, India (e-mail: dmani1982@gmail.com).

\begin{abstract}
Keywords

- COVID-19 infection

- COVID-19 testing

- day care systemic therapy

- reverse transcriptasepolymerase chain reaction

Introduction The Indian Council of Medical Research recommends coronavirus disease 2019 (COVID-19) testing for asymptomatic patients only in hospitalized condition or those seeking hospitalization for chemotherapy.

Materials and Methods This was a retrospective study to assess the incidence of asymptomatic COVID-19 positivity among asymptomatic, adult patients who were planned for systemic therapy as day care at a tertiary care cancer center. COVID-19 testing was done by reverse transcriptase-polymerase chain reaction by throat/nasopharyngeal swab once before the first cycle of systemic therapy and once every 3 weeks until completion of systemic therapy.

Results A total of 824 systemic therapies were delivered in day care from May 7 to June 20, 2020. Among the 761 COVID-19 testing done before systemic therapy, 11 patients were COVID-19 positive (1.45\%, 95\% confidence interval: 0.60-2.29). Among the 11 patients, $45 \%$ were negative before the first systemic therapy but tested positive before subsequent cycles. Among the asymptomatic COVID-19-positive patients, $54 \%$ were hospitalized for observation and $46 \%$ were on home quarantine for 14 days. None of them received investigational therapy or traditional treatment for COVID-19. None of the patients needed admission in the intensive care unit or oxygen/ventilatory support. There was no mortality among these patients. All patients turned COVID-19 negative at a median of 9 days. Currently, 45\% $(n=5 / 11)$ of the patients have resumed systemic therapy.

Conclusion The incidence of asymptomatic COVID-19 positivity among adult cancer patients planned for day care systemic therapy is low (1.45\%). Hence, COVID-19 testing should be done after informed/shared decision with the patient/attendants. Repeat COVID-19 testing before each cycle of systemic therapy is preferable, as $45 \%$ of the patients were COVID-19 negative before the first cycle of systemic therapy and turned positive before subsequent cycles in the present study. There was no morbidity or mortality in this cohort of asymptomatic COVID-19-positive cancer patients.
\end{abstract}

DOI https://doi.org/ 10.1055/s-0041-1729735 ISSN 0971-5851
(C) 2021. Indian Society of Medical and Paediatric Oncology This is an open access article published by Thieme under the terms of the Creative Commons Attribution-NonDerivative-NonCommercial-License, permitting copying and reproduction so long as the original work is given appropriate credit. Contents may not be used for commercial purposes, or adapted, remixed, transformed or built upon. (https://creativecommons.org/licenses/by-nc-nd/4.0/). Thieme Medical and Scientific Publishers Pvt. Ltd. A-12, 2nd Floor, Sector 2, Noida-201301 UP, India 


\section{Introduction}

The novel coronavirus disease 2019 (COVID-19) pandemic has caused an unprecedented situation, leading to immense stress on the health-care system across the globe. ${ }^{1}$ Patients with advanced age and comorbid illnesses such as cancer, uncontrolled diabetes, and hypertension have an increased risk of complications that can lead to mortality. ${ }^{2}$

The incidence of asymptomatic COVID-19 positivity has a high variability depending on geographic location, the number of patients tested, type of testing, etc. A study from China $(n=72,314)$ reported the lowest incidence of $1.6 \%$ and a study from Washington $(n=76)$ reported the highest incidence of $57 \%$ of asymptomatic infections. ${ }^{3}$ The infectivity of asymptomatic carriers of COVID-19 infection is low, but the communicable period could be up to 21 days. ${ }^{4.5}$ The incidence of asymptomatic COVID-19 positivity in India is currently unknown.

The American Society of Clinical Oncology (ASCO) recommends testing of asymptomatic patients for COVID-19 infection 48 to 72 hours before the initiation of immunosuppressive therapy (cytotoxic chemotherapy, stem cell transplantation, biologic therapy, cellular immunotherapy, or high-dose steroids). ${ }^{6}$

The Indian Council of Medical Research (ICMR) recommends real-time reverse transcriptase-polymerase chain reaction (RT-PCR) as the gold standard method for detecting COVID-19 infection. However, the limitation of the RT-PCR method is the need for a modern virology facility with specialized laboratory requirements. Hence, the ICMR recommends TrueNat and cartridge-based nucleic acid amplification test systems, given the widespread availability even in district and primary health-care centers. As per ICMR recommendation dated June 23,2020 , testing for asymptomatic patients is recommended for only those in hospitalized condition or those seeking hospitalization for chemotherapy. ${ }^{7}$

There is currently no recommendation for testing patients who are planned for systemic therapy on a day care basis without hospitalization. In addition, there is no recommendation to conduct repeat testing before each subsequent cycle of systemic therapy.

\section{Materials and Methods}

This was a retrospective study conducted to assess the incidence of asymptomatic COVID-19 positivity among asymptomatic, adult patients (age $\geq 18$ years) who were planned for systemic therapy on a day care basis at a tertiary care cancer center.

Patients were tested for COVID-19 by real-time RT-PCR by throat/nasopharyngeal swabs done at a government-designated COVID-19 testing center. Testing in the government hospital was done free of cost, and testing at private centers costed Rs. 3500/-per test. As per institutional guidelines, COVID-19 testing was done before the initiation of systemic therapy and once every 3 weeks during the subsequent cycles. The testing was done in the preceding 1 week before the date of systemic therapy. Patients included adults with both solid tumors and hemato-lymphoid malignancy. Pediatric patients ( $<18$ years) and adult patients who were admitted for systemic therapy were excluded from this analysis.

The systemic therapies included chemotherapy, biologics, and supportive care therapy. Patients who had COVID-19 positivity were deferred systemic therapy and referred to government-designated COVID care centers for the management of COVID-19 infection. The information about the treatment in the government-designated COVID center was obtained from the discharge summary and talking to the patient/attendants over phone. If patients presented with fever and influenza-like illness symptoms after systemic therapy, they were admitted to the severe acute respiratory infection ward for stabilization followed by COVID-19 RT-PCR testing. COVID-19-positive patients were referred to government-designated COVID centers for further management, and COVID-19-negative patients were transferred to the regular ward for further treatment.

Descriptive statistics were used to analyze the baseline characteristics.

\section{Results}

A total of 824 systemic therapies were delivered on a day care basis from May 7 to June 20 for a total period of 45 days. The number of COVID-19 testing done during the period was 761 . A total of 63 patients did not undergo COVID-19 testing before systemic therapy as they had come for either day 8 or day 15 of the scheduled regimen. Among the 761 COVID-19 testing done before systemic therapy, 11 patients reported to be COVID-19 positive $(1.45 \%, 95 \%$ confidence interval [CI]: 0.60 2.29) and 750 were COVID-19 negative (98.55\%) (-Table 1).

Among the 11 patients who were tested positive, 6 (55\%) were positive before the first cycle of systemic therapy and $5(45 \%)$ were negative before the first systemic therapy and tested positive before the subsequent cycles ( - Table $\mathbf{1}$ ).

The median age of the COVID-19-positive patients was 58 years (range: $33-74$ years). Females constituted $82 \%(n=$ $9 / 11)$ and males $18 \%(n=2 / 11)$. The most common age group affected was 50 to 59 years (45\%). The type of malignancy was a solid tumor in $82 \%(n=9 / 11)$ and hemato-lymphoid in $18 \%$ of patients $(n=2 / 11)$. Stage IV disease constituted $54 \%$ $(n=6 / 11)(-$ Table 2).

Table 1 COVID-19 test and time of testing

\begin{tabular}{|l|l|}
\hline Variable & $n(\%)$ \\
\hline COVID-19 test & $n=761$ \\
\hline Positive & $11(1.45)$ \\
\hline Negative & $750(98.55)$ \\
\hline Time of testing & $6 / 11(55)$ \\
\hline $\begin{array}{l}\text { Positive before the first systemic } \\
\text { therapy }\end{array}$ & $5 / 11(45)$ \\
\hline \multicolumn{2}{|l|}{ Positive before the subsequent cycles }
\end{tabular}

Abbreviation: COVID-19, coronavirus disease 2019. 
Table 2 Baseline characteristics of COVID-19-positive patients $(n=11)$

\begin{tabular}{|l|l|}
\hline Variable & $\boldsymbol{n}(\%)$ \\
\hline Male & $2 / 11(18)$ \\
\hline Female & $9 / 11(82)$ \\
\hline Solid malignancy & $9 / 11(82)$ \\
\hline Hematological & $2 / 11(12)$ \\
\hline Age (y) & \\
\hline $18-29$ & $0 / 11$ \\
\hline $30-39$ & $2 / 11(18)$ \\
\hline $40-49$ & $0 / 11$ \\
\hline $50-59$ & $5 / 11(45)$ \\
\hline$>60$ & $4 / 11(36)$ \\
\hline Stage IV disease & $6 / 11(54)$ \\
\hline
\end{tabular}

Abbreviation: COVID-19, coronavirus disease 2019.

Table 3 Outcome of COVID-19-positive cancer patients

\begin{tabular}{|l|l|}
\hline Outcome & $\boldsymbol{n}(\%)$ \\
\hline Recovered (COVID-19 negative) & $11(100)$ \\
\hline Ongoing infection & $0(0)$ \\
\hline Died & $0(0)$ \\
\hline Resumed systemic therapy & $5 / 11(45)$ \\
\hline
\end{tabular}

Abbreviation: COVID-19, coronavirus disease 2019.

Among the 11 asymptomatic patients diagnosed with COVID-19 positivity, 54\% $(n=6 / 11)$ were hospitalized and $46 \%$ ( $n=5 / 11)$ were on home quarantine for 14 days.

Patients were hospitalized as recommended by the government-designated COVID center for isolation and observation. None of them received any form of treatment such as investigational therapy or traditional treatment. None of the patients needed admission in the intensive care unit or oxygen/ventilatory support. There was no mortality among these patients. All patients turned COVID-19 negative at a median of 9 days. Currently, 45\% ( $n=5 / 11)$ of the patients have resumed systemic therapy as on July 8, 2020 (- Table 3).

\section{Discussion}

As on August 2, 2020, 1,750,723 individuals were diagnosed with COVID-19 infection and 37,364 (2.13\%) had died in India. ${ }^{8}$ In Tamil Nadu, 251,738 were diagnosed with COVID-19 infection and 4,034 (1.6\%) had died. ${ }^{8}$ Chennai has the highest number of COVID-19-confirmed patients $(101,951)$ in Tamil Nadu with 1,065 newly diagnosed patients, as on August 2, $2020 .^{8}$

The earlier ICMR, version 5.0 dated May 18, 2020, did not recommend COVID-19 testing for asymptomatic patients planned for chemotherapy. ${ }^{9}$ But, we did COVID-19 testing given the rapidly rising new cases in our city. However, the current study showed that COVID-19 positivity among 761 tested patients was lower than expected (1.45\%).
COVID-19 testing before systemic therapy has many benefits. First, the diagnosed patient can be deferred from the potentially harmful immunosuppressive therapy (chemotherapy/biologics/immunotherapy). Second, the patient can be referred to an infectious disease expert for evaluation, management, and isolation. Third, contact tracing of the COVID-19-positive patient can be done and all the primary contacts can undergo COVID-19 testing to rule out asymptomatic COVID-19 infection. Contact tracing and isolation have shown to be effective in breaking down the chain transmission and fattening the curve. ${ }^{10}$ Fourth, the health-care workers including the doctors, nurses, and support staff are prevented from additional exposure.

Among the 11 patients who were COVID-19 positive, 45\% ( $n=5 / 11$ ) were COVID-19 negative before the first cycle of systemic therapy and turned positive before the subsequent cycles. Currently, none of the guidelines including ASCO and ICMR recommend repeat testing in asymptomatic patients. Based on the current study, it is preferable to repeat testing before subsequent cycles as patients travel home and are at risk of exposure to COVID-19 infection.

A Chinese retrospective study on 205 cancer patients with COVID-19 infection, showed a high case fatality rate for those who had received chemotherapy within 4 weeks of the onset of symptoms. ${ }^{11}$ In a prospective observational study from the United Kingdom on 800 patients with cancer and symptomatic COVID-19 infection, the mortality was unrelated to the cytotoxic chemotherapy or anticancer therapy. ${ }^{12}$ A cohort study from the United States of America, Canada, and Spain on 928 patients with cancer and COVID-19 infection showed a mortality of $13 \%{ }^{13}$ In a study from the United Arab Emirates, 85 asymptomatic cancer patients with cancer were tested for COVID-19 infection with RT-PCR and showed that 7 patients (8.24\%) were tested positive. ${ }^{14}$

The Tata Memorial Hospital-COVID-19 working group has shown that systematic strategizing, an environment permitting healthy disagreements, rapid multipronged implementation, willingness to modify decisions at short notice, effective communication, and teamwork, is needed to manage this pandemic. ${ }^{15}$ The studies on COVID-19 infection in cancer are predominantly from China and developed countries with variable outcomes. Currently, there is a paucity of studies from India regarding the outcome of cancer patients with COVID-19 infection.

The strength of this study includes that this is the first study from India with a large number of asymptomatic patients who underwent testing. The limitation of this study is that we did not test patients who had come on day 8 or day 15 of the planned systemic therapy. The results represent the situation only during the months of May and June 2020 in Chennai. Testing leads to an additional burden on staff collecting specimens, an additional cost of personal protective equipment, a psychological burden for the patient/family if tested positive due to delay in cancer treatment and stigma surrounding quarantine, and pressure on patients to undergo testing each time before each systemic therapy. The turnaround time of COVID-19 testing would increase with increased testing. The cost of COVID-19 testing alone 
for six cycles of systemic therapy is approximately Rs. 21,000, which would double for regimens with day 8 or day 15 dose.

\section{Conclusion}

The incidence of asymptomatic COVID-19 positivity among adult cancer patients planned for day care systemic therapy is low (1.45\%, 95\% CI: 0.60-2.29). Hence, COVID-19 testing should be done after informed/shared decision with the patient/attendants. Repeat COVID-19 testing before each cycle of systemic therapy is preferable, as $45 \%$ of the patients who were COVID-19 negative before the first cycle of systemic therapy turned positive before subsequent cycles in the present study. There was no morbidity or mortality in this cohort of asymptomatic COVID-19-positive cancer patients. Further, follow-up of this study is planned given the changing dynamics of this pandemic.

\section{Acknowledgment}

We thank Dr. Gundrathi Vamsivihari and Dr. Haridas P Mani, DM Medical Oncology residents for assisting in data collection. We thank Lister Metropolis and Neuberg Diagnostics for doing the COVID-19 RT-PCR testing free of cost for patients below poverty line.

\section{Financial Support and Sponsorship \\ Nil.}

\section{Conflicts of Interest}

There are no conflicts of interest.

\section{References}

1 The Lancet Oncology. COVID-19: global consequences for oncology. Lancet Oncol 2020;21(4):46710.1016/ S1470-2045(20)30175-3

2 Wu C, Chen X, Cai Y, et al. Risk factors associated with acute respiratory distress syndrome and death in patients with coronavirus disease 2019 pneumonia in Wuhan, China. JAMA Intern Med 2020;180(7):934-943

3 Gao Z, Xu Y, Sun C, et al. A systematic review of asymptomatic infections with COVID-19. J Microbiol Immunol Infect 2020;•••Epub ahead of print [Doi: 10.1016/j. jmii. 2020.05.001]
4 Gao M, Yang L, Chen X, et al. A study on infectivity of asymptomatic SARS-CoV-2 carriers. Respir Med 2020;169:106026

$5 \mathrm{Hu} \mathrm{Z}$, Song C, Xu C, et al. Clinical characteristics of 24 asymptomatic infections with COVID-19 screened among close contacts in Nanjing, China. Sci China Life Sci 2020;63(5):706-711

6 Rappaport M. American Society of Clinical Oncology Special Report: A Guide to Cancer Care Delivery during COVID-19 pandemic. ASCO Special Report: 24 (2020). Available from: https://www.asco.org/sites/new-www.asco.org/files/content-fles/2020-ASCO-Guide-Cancer-COVID19.pdf. Accessed April 17, 2021

7 Indian Council of Medical Research. Advisory on Newer Additional Strategies for COVID-19 Testing. 1-7 (2020). Available from: http://health.delhigovt.nic.in/wps/wcm/connect/ icmr. Accessed April 17, 2021

8 Ministry of health and family welfare-Government of India FAQ. pdf. Available from: https://www.mohfw.gov.in/. Accessed April 17, 2021

9 Indian Council of Medical Research-Strategy for COVID-19 testing in India. Available from: https://www.icmr.gov.in/pdf/ covid/strategy/Testing_Strategy_v5_18052020.pdf. Accessed April 17, 2021

10 Hellewell J, Abbott S, Gimma A, et al. Centre for the Mathematical Modelling of Infectious Diseases COVID-19 Working Group. Feasibility of controlling COVID-19 outbreaks by isolation of cases and contacts. Lancet Glob Health 2020;8(4):e488-e496

11 Yang K, Sheng Y, Huang C, et al. Clinical characteristics, outcomes, and risk factors for mortality in patients with cancer and COVID-19 in Hubei, China: a multicentre, retrospective, cohort study. Lancet Oncol 2020;21(7):904-913

12 Lee LY, Cazier JB, Angelis V, et al. UK Coronavirus Monitoring Project Team. COVID-19 mortality in patients with cancer on chemotherapy or other anticancer treatments: a prospective cohort study. Lancet 2020;395(10241):1919-1926

13 Kuderer NM, Choueiri TK, Shah DP, et al. COVID-19 and Cancer Consortium. Clinical impact of COVID-19 on patients with cancer (CCC19): a cohort study. Lancet 2020;395(10241): 1907-1918

14 Al-Shamsi HO, Coomes EA, Alrawi S. Screening for COVID-19 in asymptomatic patients with cancer in a hospital in the United Arab Emirates. JAMA Oncol 2020;6(10):1627-1628 Epub ahead of print [Doi: 10.1001/jamaoncol. 2020.2548]

15 Tata Memorial Centre COVID-19 Working Group. Tata Memorial Centre COVID-19 Working Group comprises the following. The COVID-19 pandemic and the Tata Memorial Centre response. Indian J Cancer 2020;57:123-128 